\title{
EVALUACIÓN DEL BIENESTAR ANIMAL POR MEDIO DE INDICADORES CONDUCTUALES DURANTE EL SACRIFICIO DE BOVINOS
}

\author{
MARLYN H. ROMERO $P^{1}$ \\ LINA M. GONZÁLES G ${ }^{2}$ \\ CLAUDIA G. COBO A ${ }^{2}$.
}

\begin{abstract}
Recibido el 15 de agosto de 2012 y aprobado el 17 de septiembre de 2012
\end{abstract}

\section{RESUMEN}

La legislación Colombiana establece los lineamientos para el sacrificio de bovinos, el cual debe garantizar un procedimiento humanitario, además de cumplir con ciertos parámetros de calidad del producto final. El objetivo del estudio fue evaluar la eficiencia en la insensibilización en dos plantas de sacrificio como indicador de bienestar animal. Se evaluó la insensibilización de 1343 bovinos. Como variables se emplearon los signos de pérdida de sensibilidad (reflejo corneal, intentos de incorporarse, vocalizaciones y respiración rítmica) e indicadores conductuales de pérdida de Bienestar Animal (BA) (resbalones, caídas, vocalizaciones, saltos y marcha hacia atrás). Se midió el tiempo de retraso en la insensibilización, el número de disparos por animal, la localización correcta del impacto y el tiempo entre insensibilización y sangría. El $96.5 \%$ de los bovinos colapsaron inmediatamente después de recibir el primer impacto, sin embargo, el 23.6\% ( $n=307)$ de éstos recuperó la sensibilidad antes de la sangría, debido al amplio intervalo de tiempo entre la insensibilización y la sangría superior a los 60 segundos $(98.7 \%)$. Se encontró asociación significativa entre el retraso en la insensibilización y el número de eventos conductuales $(p<0.01)$. Asimismo, se hallaron diferencias para los indicadores conductuales entre las plantas de sacrificio $(p<0.01)$, encontrándose mayor incidencia en la planta A, la cual contaba con un método de sujeción de cabeza en el cajón de insensibilización, pero no de cuerpo. De otra parte, se observaron malas prácticas de manejo y conducción de los animales en la planta B como sujetar y empujar el animal con la picana eléctrica. Se concluye que en las plantas evaluadas el bienestar animal durante el sacrificio de bovinos es deficiente, se hace necesario la capacitación del personal, mejoras en la infraestructura y la implementación de indicadores de evaluación. 


\title{
PALABRAS CLAVE:
}

Bienestar del animal, comportamiento, ganado de carne, insensibilización, sacrificio humanitario.

\section{ASSESSMENT OF ANIMAL WELFARE USING BEHAVIORAL INDICATORS DURING CATTLE SLAUGHTER}

\begin{abstract}
Colombian laws establish the guidelines for slaughtering of cattle which have to $f$ guarantee a humane procedure, besides complying with some quality parameters for the final product. The objective of this study was to evaluate efficiency of the stunning process in two slaughterhouses as an indicator of animal welfare. Stunning was evaluated in 1343 bovines. Signs of loss of consciousness (corneal reflex, attempts to head up, vocalizations and rhythmic breathing) as well as behavioral indicators of Animal Welfare (AW) loss (slipping, falling, vocalizations, jumping and backward movements) were assessed to identify animal welfare standards. Besides, the stunning delay, the number of shots per animal, the appropriate location of the shots and the interval between shot and bleeding were measured. Although, $98.5 \%$ of animals collapsed immediately after receiving the first shot, $23.6 \% \quad(n=307)$ recovered sensitivity before bleeding, because of the wide interval between stunning and bleeding which was higher than 60 seconds (98.7\%). There was a significant association between stunning delay and the number of behavioral events counted $(p<0.01)$. Moreover, differences in the behavioral indicators among the slaughterhouses evaluated were found $(p<0.01)$ showing greater incidence in slaughter house " $A$ " which had a head fixation system, but not one to hold still the entire body of the animal. Aversive behavioral responses were encountered in plant "B", such as the use of cattle prods to hold and push the animal while it was in the stunning box. The results indicate that in the slaughterhouses evaluated animal welfare during slaughtering is defficient and it is necessary to implement personnel training, improvement of infrastructure and evaluation indicators.
\end{abstract}

\section{KEY WORDS:}

Animal welfare, behavior, livestock, stunning, humanae slaughtering 


\section{INTRODUCCIÓN}

El sacrificio (insensibilización y sangría) de los animales de abasto público, es considerada una etapa crítica que afecta el bienestar animal y la calidad e inocuidad de la carne (Ferguson \& Warner, 2008). En Colombia, la legislación sanitaria exige que el sacrificio de bovinos se realice bajo lineamientos humanitarios (Ministerio de la Protección Social, 2007b). Sin embargo, existe poca preocupación y conciencia por evitar el sufrimiento de los bovinos en esta etapa, aduciendo que el animal morirá en pocos minutos, aspecto que ha sido descrito en otros países del continente (Gallo, 2010). Desde el punto de vista del bienestar animal, el sacrificio tiene como finalidad evitar el sufrimiento y estrés innecesario a los animales al momento de provocarles la muerte (Organización Mundial de Sanidad Animal, 2012), mediante el uso de sistemas de insensibilización que garanticen la pérdida de conciencia instantáneamente, y se mantenga tal estado hasta la muerte (Ministerio de la Protección Social, 2007a, 2007b). Con relación a la calidad de la carne, las malas prácticas de sacrificio, en especial la insensibilización, pueden favorecer la presencia de hemorragias petequiales y equimóticas en músculos, así como la fractura de huesos, que conllevan a disminuir el valor comercial de los cortes (Gregory, 2005; Grandin, 2006; Linares, Bornez, \& Vergara, 2007). En lo referente a la inocuidad, la carne proveniente de animales mal sangrados, favorece el crecimiento microbiano y disminuye su vida útil (Blokhuis, Keeking, Gavinelli y Serratosa, 2008). A pesar de estas implicaciones, son frecuentes los métodos no aceptados para insensibilizar a los bovinos y las malas prácticas de manejo. Por otra parte, se encuentra una baja eficacia de los métodos, falta de capacitación y entrenamiento del personal responsable y ausencia de indicadores que permitan evaluar objetivamente el proceso (Gallo, 2007; Romero \& Sánchez, 2011).

Para evaluar la efectividad de la insensibilización y la sangría se han utilizado indicadores conductuales o basados en el animal. Estos indicadores han sido implementados por el Departamento de Agricultura de los Estados Unidos (USDA), como soporte a las actividades de inspección, vigilancia y control en los frigoríficos de dicho país. Asimismo, multinacionales procesadoras de alimentos como MacDonald's y Wendy's, seleccionan sus proveedores de carne de acuerdo con estos lineamientos (Grandin, 2010a). La importancia del uso de indicadores conductuales radica en que miden condiciones que están relacionadas con malas prácticas de manejo, equipos mal diseñados o comportamientos abusivos por parte de los manejadores hacia los animales (Sejian, Lakritz, Ezeji \& 
Lal, 2011). El uso de estos indicadores durante el sacrificio de animales de abasto es una práctica inusual en Colombia, que podría ser un soporte para las autoridades sanitarias, para evaluar el impacto de las prácticas habituales de las plantas de sacrificio comercial sobre el bienestar, la calidad e inocuidad de la carne. Su implementación se recomienda porque se basan en el conocimiento científico, permiten conocer la tendencia de la medición en el tiempo, hacen viable la medición bajo condiciones comerciales y suministran información sobre posibles problemas de bienestar animal y sus causas (Sejian, et al. 2011). El objetivo del presente trabajo consistió en evaluar mediante indicadores conductuales la eficiencia en la insensibilización en dos plantas de sacrificio comercial localizadas en el occidente colombiano.

\section{METODOLOGÍA}

Se realizó un estudio de corte transversal en dos plantas de sacrificio comercial autorizadas por el INVIMA, con un promedio mensual de sacrificio de 900 bovinos. Las dos plantas tenían cajones de insensibilización exclusivos para bovinos, construidos en cemento y provistos de puertas de entrada de guillotina. La planta $A$, contaba con un cajón de insensibilización provisto de un sistema de sujeción de cabeza, pero sin sujeción de cuerpo, mientras que la planta $\mathrm{B}$, sin sujeción de cabeza y cuerpo (Figura 1). Se utilizaron en los dos casos pistolas de proyectil retenido con penetración de cráneo, impulsadas mediante aire comprimido, las cuales funcionan a una presión promedio de 12 bar constante. La planta A tenía instaurado un protocolo para el mantenimiento y limpieza rutinaria de la pistola, mientras que la planta $B$, carecía de éste.

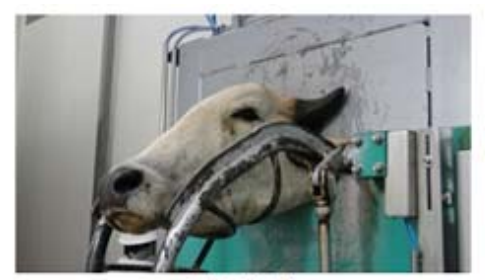

Planta A

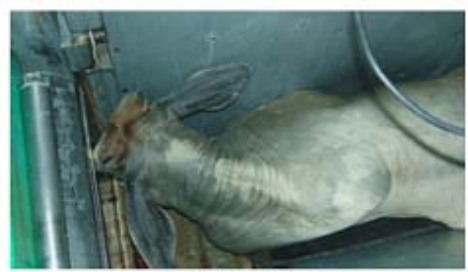

Planta B

Figura 1. Planta A. Sistema de insensibilización con sujeción de cabeza

solamente. Planta B. Cajón de insensibilización, sin sujeción de cabeza, ni

cuerpo

Indicadores evaluados. De acuerdo con Grandin (2010a), se seleccionaron aleatoriamente 1343 bovinos; 771 en la planta A y 572 en la planta B, en diferentes días y turnos del proceso. Las observaciones conductuales se realizaron en el cajón de insensibilización registrándose el número de 
eventos: resbalones, caídas, vocalizaciones, marcha hacia atrás y uso de picana eléctrica. Para la evaluación de tales indicadores, se utilizaron como criterios aceptables los propuestos por el Instituto americano de la carne y el comité ético del bienestar animal (Grandin, 2010b); donde se exigen porcentajes $<3 \%$ para las caídas, $<1 \%$ para los resbalones, $<3 \%$ para vocalizaciones y $<25 \%$ para la aplicación de picana eléctrica en el cajón de insensibilización. Asimismo, se contabilizó el número de animales que permanecieron insensibles durante el izado, mediante la evaluación de la presencia/ausencia de los signos: reflejo corneal, intento de incorporarse, vocalizaciones y respiración rítmica.

Se estudiaron indicadores administrativos como la efectividad de la insensibilización (\% de animales insensibilizados en el primer intento), el retraso en la insensibilización (intervalo de tiempo entre la entrada del animal al cajón de insensibilización y el momento en el que recibe el primer disparo), localización del disparo en el lugar correcto e intervalo entre el primer disparo y la sangría (Grandin, 2010c; Bourguet, Deiss, Boissy, Andanson, \& Terlouw, 2011). Adicionalmente, mediante observación directa se evaluaron las prácticas de manejo de los operarios durante el ingreso de los animales en el cajón de insensibilización, la sincronización de actividades entre el personal, el uso de indicadores de evaluación, la actitud del personal frente a los bovinos y las tareas específicas del personal en cada turno de trabajo.

Análisis estadístico. La información se analizó mediante el programa Stata Versión 12.0 (College Station, Texas, $\mathrm{EU})$. Se presentaron los promedios \pm desviaciones estándar de los indicadores evaluados. Se estudiaron las correlaciones entre las variables mediante los coeficientes de Pearson, así como las diferencias entre las dos plantas mediante la prueba de $\mathrm{Chi}^{2}$, con un nivel de significancia del $95 \%$.

\section{RESULTADOS}

Los indicadores conductuales evidencian prácticas de bienestar inaceptables durante el ingreso de los bovinos al cajón de insensibilización en las dos plantas (Tabla 1). Se observaron diferencias significativas en la proporción de resbaladas y la marcha hacia atrás entre las dos plantas, siendo más frecuentes en la planta $B(p>0.05)$. 
Tabla 1. Porcentaje de eventos conductuales de los bovinos evaluados en el cajón de insensibilización.

\begin{tabular}{|c|c|c|c|c|c|}
\hline Variable & $\begin{array}{c}\text { Categoría } \\
\text { (\# } \\
\text { eventos) }\end{array}$ & $\begin{array}{c}\text { Planta } A \mathrm{n}=771 \\
\mathrm{n}(\%)\end{array}$ & $\begin{array}{l}\text { Media } \pm \text { DS } \\
\text { presencia } \%\end{array}$ & $\begin{array}{c}\text { Planta B n=572 } \\
n(\%)\end{array}$ & $\begin{array}{l}\text { Media } \pm \text { DS } \\
\text { presencia } \%\end{array}$ \\
\hline Resbalarse & & & $0.57 \pm 1.14$ & & $0.28 \pm 0.72$ \\
\hline \multirow[t]{7}{*}{$\mathrm{Si}$} & & & 30.6 & & 17.1 \\
\hline & 0 & $535(69.4)$ & & $474(82.9)$ & \\
\hline & 1 & $119(15.4)$ & & $50(8.7)$ & \\
\hline & 2 & $74(9.6)$ & & $37(6.5)$ & \\
\hline & 3 & $21(2.8)$ & & $9(1.5)$ & \\
\hline & 4 & $11(1.4)$ & & $1(0.2)$ & \\
\hline & 25 & $11(1.4)$ & & $1(0.2)$ & \\
\hline Vocalizaciones & & & $0.13 \pm 0.44$ & & $0.15 \pm 0.45$ \\
\hline \multirow{6}{*}{ Si } & & & 9.8 & & 12.1 \\
\hline & 0 & $695(90.2)$ & & $503(87.9)$ & \\
\hline & 1 & $55(7.1)$ & & $53(9.3)$ & \\
\hline & 2 & $17(2.2)$ & & $14(2.4)$ & \\
\hline & 3 & $3(0.4)$ & & $1(0.2)$ & \\
\hline & 4 & $1(0.1)$ & & $1(0.2)$ & \\
\hline \multirow{6}{*}{$\begin{array}{c}\text { Caidas } \\
\text { Si }\end{array}$} & & & $0.10 \pm 0.32$ & & $0.09 \pm 0.30$ \\
\hline & & & 9.6 & & 9.8 \\
\hline & 0 & $697(90.4)$ & & $516(90.2)$ & \\
\hline & 1 & $71(9.2)$ & & $55(9.6)$ & \\
\hline & 2 & $2(0.3)$ & & $1(0.2)$ & \\
\hline & 3 & $1(0.1)$ & & 0 & \\
\hline \multirow[t]{3}{*}{$\begin{array}{l}\text { Marcha hacia atrás } \\
\text { Si }\end{array}$} & & & $\begin{array}{c}0.07 \pm 0.26 \\
7.2\end{array}$ & & $\begin{array}{c}0.20 \pm 0.40 \\
20.3\end{array}$ \\
\hline & No & $711(92.2)$ & & $456(79.7)$ & \\
\hline & $\mathrm{Si}$ & $60(7.8)$ & & $116(20.3)$ & \\
\hline
\end{tabular}

La planta A no utiliza tábano o picana eléctrica en el cajón de insensibilización. La planta B ocasionalmente la usa para inmovilizar los bovinos, lo cual corresponde a la restricción del movimiento de los animales al ser presionados contra las paredes del cajón de insensibilización, y de esta forma lograr la aplicación del disparo en el sitio correcto $(0.8 \%, n=5 / 572)$. Es habitual utilizarla para empujar el cuerpo del bovino con la finalidad de facilitar su salida del cajón de insensibilización (38.9\%, $\mathrm{n}=223 / 572$ ). Adicionalmente, la puerta de guillotina localizada a la entrada del cajón de insensibilización, es usada en las dos plantas para golpear a los animales y acelerar su ingreso al cajón, siendo más frecuente y estadísticamente significativa esta práctica en la planta $\mathrm{B}$ $(62.8 \%)$, que en la planta A $(36.8 \%)(p<0.05)$.

Con relación a la efectividad de la insensibilización, el signo de retorno a la sensibilidad prevalente fue la respiración rítmica, observándose con mayor frecuencia en la planta $B$ (20.1\%) (Tabla 2). En general se observaron diferencias significativas en estas variables al comparar las proporciones observadas en las dos plantas $(p<0.05)$. 
Tabla 2. Presencia de signos indicadores de sensibilidad durante el izado en las dos plantas de sacrificio evaluadas.

\begin{tabular}{ccccc}
\hline Signos de sensibilidad & $\begin{array}{c}\text { Observaciones } \\
(\mathbf{n})\end{array}$ & $\begin{array}{c}\text { Planta A } \\
\mathbf{n}(\%)\end{array}$ & $\begin{array}{c}\text { Observaciones } \\
(\mathbf{n})\end{array}$ & $\begin{array}{c}\text { Planta B } \\
\mathbf{n}(\%)\end{array}$ \\
\hline Colapso & 771 & $770(99.9)$ & 572 & $528(92.3)$ \\
Reflejo corneal & 756 & $4(0.5)$ & 572 & $11(1.9)$ \\
Intento incorporarse & 771 & $5(0.6)$ & 572 & $23(4)$ \\
Vocalizaciones & 771 & 0 & 572 & $7(1.2)$ \\
Respiración Rítmica & 769 & $103(13.4)$ & 572 & $115(20.1)$ \\
Movimiento Cola & 771 & $102(13.2)$ & 542 & $47(8.6)$ \\
\hline
\end{tabular}

Las dos plantas presentaron intervalos prolongados entre la insensibilización y la sangría, superiores a los 60 segundos (Ministerio de la Protección Social, 2007b) representando una mayor problemática para la planta A (Tabla 3). El retraso en la insensibilización presentó un rango entre 2 y $320 \mathrm{~s}$, el cual tuvo asociación significativa con el número de eventos conductuales observados en el cajón de insensibilización $(p<0.05)$.

Tabla 3. Resumen de los indicadores administrativos en las dos plantas evaluadas.

\begin{tabular}{|c|c|c|c|c|c|}
\hline Variable & Categoria & $\begin{array}{c}\text { Observaciones } \\
\text { (n) }\end{array}$ & $\begin{array}{c}\text { Planta } A \mathrm{n}=755 \\
\mathrm{n}(\%)\end{array}$ & $\begin{array}{c}\text { Observaciones } \\
\text { (n) }\end{array}$ & $\begin{array}{c}\text { Planta B } \\
\mathrm{n}(\%)\end{array}$ \\
\hline $\begin{array}{c}\text { Insensibilización } \\
\text { efectiva }\end{array}$ & $\begin{array}{r}\text { No } \\
\text { Si }\end{array}$ & 755 & $\begin{array}{l}164(21.7) \\
591(78.3)\end{array}$ & 542 & $\begin{array}{l}143(26.4) \\
399(73.6)\end{array}$ \\
\hline $\begin{array}{l}\mathrm{N}^{2} \text { de disparos por } \\
\text { animal }\end{array}$ & $\begin{array}{l}1 \\
2 \\
3 \\
4\end{array}$ & 771 & $\begin{array}{c}770(99.9) \\
1(0.1) \\
0 \\
0\end{array}$ & 572 & $\begin{array}{c}521(91.1) \\
35(6.1) \\
15(2.6) \\
1(0.2)\end{array}$ \\
\hline $\begin{array}{c}\text { Disparo en el lugar } \\
\text { correcto }\end{array}$ & $\begin{array}{l}\mathrm{Si} \\
\text { No }\end{array}$ & 771 & $\begin{array}{c}771(100) \\
0\end{array}$ & 572 & $\begin{array}{c}531(92.9) \\
41(7.1)\end{array}$ \\
\hline $\begin{array}{l}\text { Retraso de la } \\
\text { insensibilización (s) }\end{array}$ & $\begin{array}{l}\leq 60 \\
>60\end{array}$ & 771 & $\begin{array}{c}746(96.7) \\
25(3.3)\end{array}$ & 572 & $\begin{array}{c}536(93.7) \\
36(6.3)\end{array}$ \\
\hline $\begin{array}{l}\text { Intervalo entre el primer } \\
\text { disparo y la sangría (s) }\end{array}$ & $\begin{array}{c}\leq 60 \\
61-120 \\
121-180 \\
181-240 \\
\geq 240\end{array}$ & 288 & $\begin{array}{c}1(0.3) \\
102(35.4) \\
172(59.8) \\
12(4.2) \\
1(0.3)\end{array}$ & 204 & $\begin{array}{c}5(2.5) \\
157(77) \\
40(19.7) \\
1(0.4) \\
1(0.4)\end{array}$ \\
\hline
\end{tabular}

\section{DISCUSIÓN}

Durante el faenado los bovinos son sometidos a factores estresantes que generan cambios comportamentales, dichos factores están relacionados con la interacción manejador-animal y con la infraestructura y diseño de las plantas (Grignard, Boivin, Boissy \& Neindre, 2001; Grandin, 2010b). En el presente estudio fueron frecuentes las resbaladas y las caídas durante el ingreso al cajón de insensibilización en las dos plantas estudiadas (Tabla 1), aspectos que incrementan el estrés de los animales y aumentan el riesgo de la presencia de contusiones y carnes de menor calidad (Miranda-de la Lama, Rivero, Chacón, \& García-Belenguer, 2010; Strappini, Frankena, Metz, Gallo \& Kemp, 2012). Estas conductas del ganado 
están relacionadas con pisos deslizantes y mal diseño del cajón de insensibilización (Grandin, 2003; Grandin, 2006). De igual forma, la marcha hacia atrás o intento de devolverse de los bovinos, se debe al mal diseño de los corredores de conducción, al ruido generado por los equipos neumáticos del área de sacrificio, a la falta de iluminación del cajón de insensibilización y a las conductas aversivas del personal durante el arreo (Grandin, 2006; Miranda-de la Lama, et al. 2010). Teniendo en cuenta que en la actualidad las plantas de sacrificio colombianas se encuentran en proceso de remodelación de su infraestructura acorde con las exigencias reglamentarias (Ministerio de la Protección Social, 2007a, 2007b), es primordial que diseñen sus instalaciones con mangas de conducción sólidas y curvas, pisos antideslizantes y que eviten acúmulos de agua, sistemas de aislamiento de ruido de sus instalaciones, cajones de insensibilización con pisos antideslizantes, de dimensiones acordes con el tamaño del ganado colombiano, iluminados y provistos de una rampa para la recepción del ganado insensibilizado, entre otros aspectos (Romero \& Sánchez, 2011).

La relación manejador-animal inapropiada hace al ganado más reactivo al hombre, incrementa el miedo, el estrés y la frecuencia de vocalizaciones, resbalones y caídas, lo cual repercute en la eficiencia del proceso, aumenta el riesgo de contusiones y de accidentes de trabajo (Minka \& Ayo, 2007; Paranhos da Costa, Huertas, Gallo \& Dalla Costa, 2012). En la planta $B$ se evidenció el uso excesivo de tábano o picana eléctrica con fines de restricción de movimiento $(0.8 \%)$, lo cual se considera inaceptable, así como, para ayudar a la conducción del ganado (38.9\%), está última por encima de los criterios considerados como excelentes ( 5\%) (Grandin, 2010b). Adicionalmente, durante el estudio se observó la aplicación del tábano en áreas sensibles del animal como ojos, cara y genitales, lo cual representa una práctica abusiva para la Organización Mundial de Sanidad Animal-OIE-(2012) y que es considerada como una causa que produce la pérdida de la auditoria de bienestar animal en las plantas de sacrificio de los Estados Unidos (Grandin, 2010a; Grandin, 2010c). El uso de la puerta de guillotina para golpear a los bovinos e incentivar su ingreso al cajón, observada en las dos plantas evaluadas, es una práctica considera como abusiva, y representa un riesgo potencial para la presencia de contusiones en el lomo generando pérdidas económicas importantes a la industria cárnica (Strappini, et al. 2012).

Las expresiones acústicas o vocalizaciones son un indicador del estatus de bienestar animal fácil de medir y eficiente para identificar problemas de los equipos o de manejo inapropiado (Manteuffel, Puppe, Schön \& Vergara, 
2004; Grandin 2010c). La prevalencia de vocalizaciones en el cajón de insensibilización en las plantas evaluadas superó los niveles de puntuación aceptables (3-5\%) (Grandin, 2010b) (Tabla 1). El incremento de vocalizaciones durante la movilización ha sido relacionado con el uso excesivo de tábano eléctrico, los resbalones y las caídas. Durante la insensibilización con problemas del equipo, calibración, mantenimiento, capacitación del personal, animales muy nerviosos, cartuchos húmedos, recuperación de la sensibilidad y la presión excesiva del sujetador de cabezas (Grandin, 2001), factores que estuvieron relacionados en la presente investigación.

A pesar de que en las dos plantas se observó en alta proporción el colapso de los animales con el primer impacto de la pistola durante el noqueo (A: $99.9 \%$ y B: $92.3 \%$ ) (Tabla 2), no se evidenció concordancia con la insensibilización efectiva, porque se presentaron signos de retorno a la sensibilidad durante el izado (Tablas 2 y 3 ), aspecto previamente reportado por otros investigadores (Gregory, Lee \& Widdicombe, 2007). El signo de sensibilidad más frecuente fue la respiración rítmica, considerado como un indicador fiable para evaluar la sensibilidad de los animales, pues manifiesta que la función medular ha sido comprometida de forma incompleta, retrasándose la falla cardiopulmonar y generando un mayor riesgo para que los bovinos recuperen la conciencia (Gregory, et al. 2007). Se considera que la presencia de un sólo signo de sensibilidad no puede ser concluyente para establecer la inconciencia de los bovinos, la detección de varios de ellos en alta proporción, refleja una baja eficacia en este aspecto y por ende no se está logrando el objetivo primordial de la insensibilización, que es evitar el sufrimiento de los animales (Grandin, 2006), aspecto que fue detectado en el presente estudio (Tabla 2). Otro signo que demostró la ineficacia de esta etapa, fue el intento de incorporarse o de levantar la cabeza en el riel de sangría, siendo catalogado como uno de los indicadores más fiables de retorno a la sensibilidad (Grandin, 2010c). A pesar de que la planta A contaba con un sistema de sujeción de cabeza y con un protocolo para el mantenimiento preventivo de la pistola, la efectividad de la insensibilización no superó los límites aceptables (90-94\%) (Gallo, Teuber, Cartes, Uribe \& Grandin, 2003; Grandin, 2010a).

Respecto al intervalo de tiempo entre el primer disparo y la sangría se hallaron tiempos superiores a 60 segundos en las dos plantas (A: $99.7 \%$ y B: $97.5 \%$ ), lo cual favorece la recuperación de la conciencia de los animales y el sufrimiento durante la sangría. Los factores que contribuyeron a retrasar el inicio de la sangría fueron: falta de coordinación entre los operarios responsables de la 
insensibilización y la sangría, ingreso prematuro de los animales al cajón de insensibilización con el ánimo de acelerar la eficiencia de la faena, dificultad de expulsar los animales del cajón de insensibilización por fallas de diseño, ausencia de un protocolo que estandarice estas etapas, falta de auditoría interna, entrenamiento y capacitación del personal, falta de sensibilidad del personal por el sufrimiento animal además de cansancio del personal por sobrecarga de funciones, especialmente en la planta B, donde el personal participa en el beneficio de porcinos y en las labores de higiene y desinfección de las instalaciones. Estos problemas han sido identificados también por otros investigadores (Gallo, et al. 2003; Bourguet, et al. 2011; Miranda-de la Lama, et al. 2012).

Se concluye que en las plantas evaluadas se presentan problemas graves de bienestar animal durante el sacrificio, al compararlos con algunos estándares internacionales altamente valorados y estandarizados (Grandin, 2010b). Según las observaciones conducidas, se podrían relacionar con problemas de diseño de las instalaciones, falta de capacitación y sensibilización del personal sobre lineamientos de bienestar animal, ausencia de indicadores para auditar esta etapa y falta de compromiso gerencial para implementar criterios de evaluación. Se requiere por tanto, fortalecer el conocimiento de las implicaciones del bienestar animal en la calidad, inocuidad y productividad de la cadena cárnica bovina y la implementación de indicadores conductuales para identificar los puntos críticos durante esta etapa.

\section{AGRADECIMIENTOS}

Los autores quieren expresar sus agradecimientos a la Vicerrectoría de Investigaciones de la Universidad de Caldas y a COLCIENCIAS (Proyecto Código 1127-48925244) por la financiación.

\section{BIBLIOGRAFÍA}

- Blokhuis, H.J.; Keeling, L.J.; Gavinelli, A.; Serratosa, J. (2008). Animal welfare's impact on the food chain. Trends in Food Science \& Technology. 19: S79-S87.

- Bourguet, C.; Deiss, V.; Boissy, A.; Andanson, S. \& Terlouw, E. M. C. (2011). Effects of feed deprivation on behavioral reactivity and physiological status in Holstein cattle. Journal of Animal Science. 88: 3272-3285. 
- Ferguson, D.M. \& Warner, R.D. (2008). Have we underestimated the impact of pre-slaughter stress on meat quality in ruminants? Meat Science. 80: 12-19.

- Gallo, C. (2007). Animal welfare in the Americas. Compendium of technical items presented to the international committee or to the regional commissions of the OIE. Florianopolis, Brasil.

- Gallo, C. (2010). Bienestar animal y buenas prácticas de manejo animal relacionadas con la calidad de la carne. En: Bianchi, G. \& Feed, O. (Eds.). Introducción a la ciencia de la carne. Uruguay., pp. 455-494.

- Gallo, C.; Teuber, C.; Cartes, M.; Uribe, H. \& Grandin, T. (2003). Mejoras en la insensibilización de bovinos con pistola neumática de proyectil retenido tras cambios de equipamiento y capacitación del personal. Archivos de Medicina Veterinaria. 35:159-170.

- Grandin, T. (2001). Cattle vocalizations are associated with handling and equipment problems at beef slaughter plants. Applied Animal Behavior Science. 71: 191-201.

- Grandin, T. (2003).Transferring results of behavioral research to industry to improve animal welfare on the farm, ranch and the slaughter plant. Applied Animal Behavior Science. 81: 215-228.

- Grandin,T. (2006). Progress and challenges in animal handling and slaughter in the U.S. Applied Animal BehaviorScience.100:129-139.

- Grandin, T. (2010a). Auditing animal welfare at slaughter plants. Meat Science. 86, 56-65.

- Grandin, T. (2010b). Recommended animal handling guidelines audit guide: A systematic approach to animal welfare. Obtenido el 20 de agosto de 2011. Desde http://www.animalhandling.org/ht/a/GetDocumentAction/i/5 8425.

- Grandin, T. (2010c). Slaughter plants: behavior and welfare assessment. En: Encyclopedia of Animal Behavior. Colorado: Elsevier., pp.197-508

- Gregory, N.G. (2005). Recent concerns about stunning and slaughter. Meat Science. 70: 481-491.

- Gregory, N.G.; Lee, C. J. \& Widdicombe, J.P. (2007). Depth of concussion in cattle shot by penetrating captive bolt.Meat Science. 77: 499-503.

- Grignard, L.; Boivin, X.; Boissy, A. \& Neindre, P.L. (2001). Do beef cattle react consistently to different handling situations?.Applied Animal Behavior Science. 71: 263-276.

- Linares, M.B.; Bornez, R. \& Vergara, H. (2007). Effect of different stunning systems on meat quality of light lamb. Meat Science. 76: 657- 681.

- Manteuffel, G.; Puppe, B. \& Schön, P. (2004). Vocalization of farm animals as a measure of welfare. Applied Animal Behavior Science.88: 163-182.

- Ministerio de la Protección Social. (2007a). Decreto 1500. Bogotá, Colombia. 
- Ministerio de la Protección Social. (2007b). Resolución 2905. Bogotá, Colombia.

- Minka, N.S. \& Ayo, J.O. (2007). Effects of loading behavior and road transport stress on traumatic injuries in cattle transported by road during the hot-dry season. Livestock Science. 107: 91-95.

- Miranda-de la Lama, G.; Leyva, I.G.; Barrera-Serrano, A.; Pérez-Linares, C.; Sánchez-López, E.; Maria, G. \& Figueroa-Saavedra, F. (2012). Assessment of cattle welfare at a commercial slaughter plant in the northwest of Mexico. Tropical Animal Health Production. 44: 497-504.

- Miranda-de la Lama, G.C.; Rivero, L.; Chacón, G.; GarciaBelenguer, S. (2010). Effect of the pre-slaughter logistic on some indicators of welfare in lambs. Livestock Science.128: 52-59.

- Organización mundial de Sanidad Animal-OIE- (2012). Terrestrial animal health code. Obtenido el 14 de agosto de 2012. Desde http://www.oie.int/en/international-standardsetting/terrestrial-codelaccess-onlinel.

- Paranhos da Costa, M.J.; Huertas, S.M.; Gallo, C. \& Dalla Costa, O.A. (2012). Strategies to promote farm animal welfare in Latin America and their effects on carcass and meat quality traits. Meat Science. 92: 221-226.

- Romero, M.H., \& Sánchez, J.A. (2011). Implicaciones de la inclusión del bienestar animal en la legislación sanitaria colombiana. Revista Colombiana de Ciencias Pecuarias. 24: 93-101.

- Sejian, V.; Lakritz, J.; Ezeji, T. \& Lal, R. (2011). Assessment methods and indicators of animal welfare.Asian Journal of Animal and Veterinary Advances. 6: 301-315.

- Strappini, A.C.; Frankena, K.; Metz, J.H.M.; Gallo, C. \& Kemp, B. (2012). Characteristics of bruises in carcasses of cows sourced from farms or from livestock markets. Animal. 6: 502-509.

1. MVZ, Esp., Msc., Candidata a Doctorado. Profesora Departamento de Salud Animal, Grupo de Investigación CIENVET, Facultad de Ciencias Agropecuarias, Universidad de Caldas, Manizales, Colombia. E-mail: marlyn.romero@ucaldas.edu.co

2. MVZ, Joven Investigadora Grupo CIENVET, Facultad de Ciencias Agropecuarias, Universidad de Caldas, Manizales, Colombia. E-mail: linago14@gmail.com, claugis@hotmail.com 